\title{
Molecular modeling study of uncharged oximes compared to HI-6 and 2-PAM inside human AChE sarin and VX conjugates
}

Felipe Rodrigues de Souza ${ }^{a, c}$, Danielle Rodrigues Garciaa, Teobaldo Cuya ${ }^{b}$, André Silva Pimentel ${ }^{c}$, Arlan da Silva Goncalves ${ }^{d}$, Ricardo Bicca de Alencastro ${ }^{e}$ and Tanos Celmar Costa França $a^{a, f, *}$

aLaboratory of Molecular Modeling Applied to Chemical and Biological Defense

(LMCBD) Military Institute of Engineering, Rio de Janeiro/RJ, Brazil;

bFaculty of Technology, University of the State of Rio de Janeiro, Resende/RJ, Brazil

'Department of Chemistry, Pontifical Catholic University of Rio de Janeiro, Rio de Janeiro/RJ, Brazil;

${ }^{d}$ Federal Institute of Education Science and Technology of Espirito Santo, Unit Vila Velha, Vila Velha/ES, Brazil

eFederal University of Rio de Janeiro, Chemistry Institute, Rio de Janeiro/RJ, Brazil.

fDepartment of Chemistry, Faculty of Science, University of Hradec Kralove, Rokitanskeho 62, 50003 Hradec Kralove, Czech Republic.

\section{Supplementary Material}




\section{Oximes ionization constant}

The study of the oxime ionization constant showed that HI-6, RS41A, and $\mathrm{RS194B}$ present ionization in an environment with physiological $\mathrm{pH}(\mathrm{pH}=7.4)$ as shown in Figure $\mathrm{S} 1$ below while the other oximes did not present ionization. HI-6 shows a deprotonation of oxime $\mathrm{OH}$, with a prevalence of $93 \%$, while $\mathrm{RS} 41 \mathrm{~A}$ and RS194B shows the cyclic $\mathrm{N}$ atom protonated, with prevalence of $96 \%$ and $98 \%$, respectively. The oxime structures at $\mathrm{pH}=7.4$ were used for molecular modeling studies, since this is the blood $\mathrm{pH}$ in the way towards the CNS.

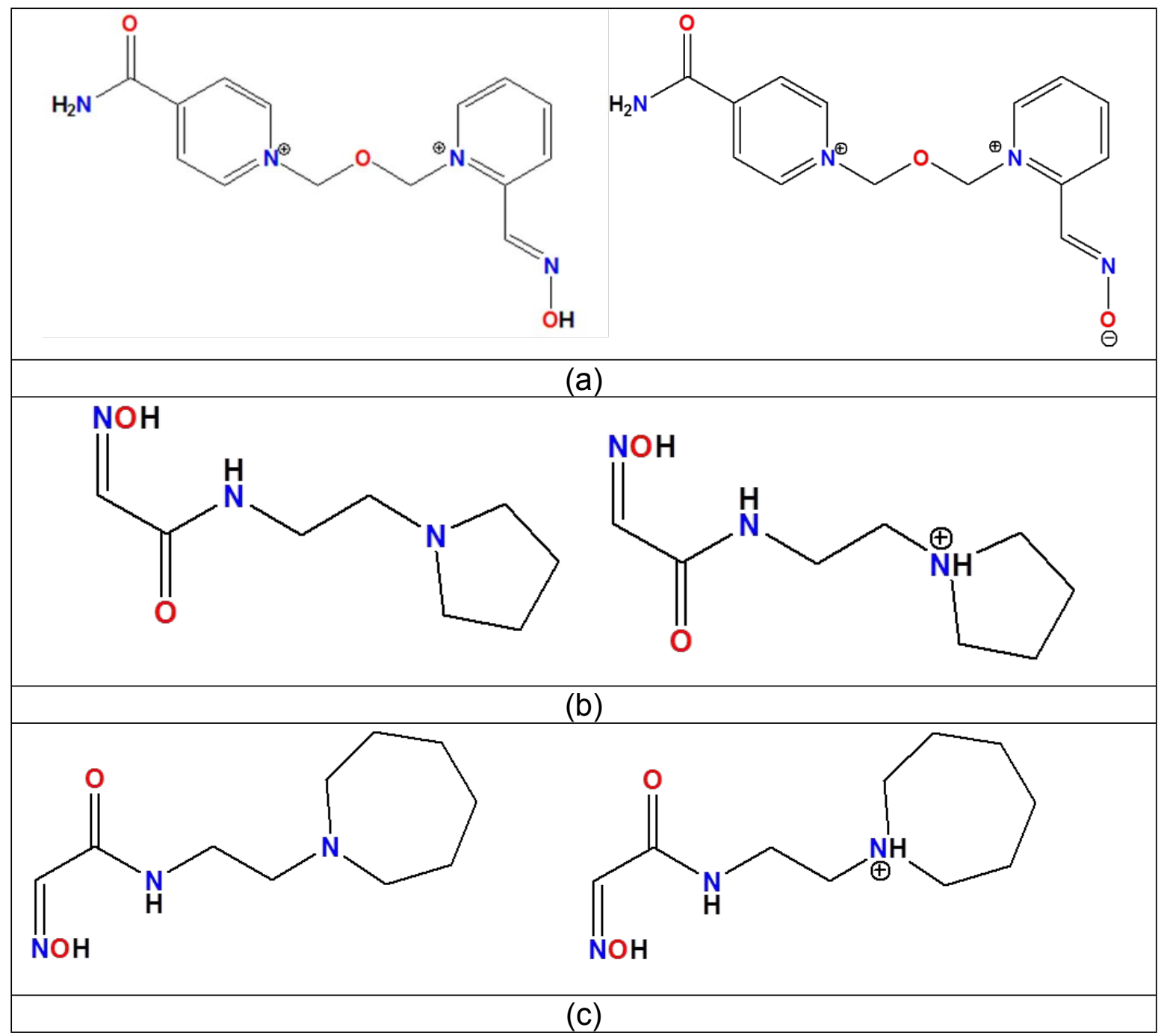

Figure S1) Structures at $\mathrm{pH}<7$ (right) and $\mathrm{pH}=7,4$ (left) of oximes (a) $\mathrm{HI}-6$ (b) RS41A and (c) RS194B. 


\section{Oxime interaction modes}

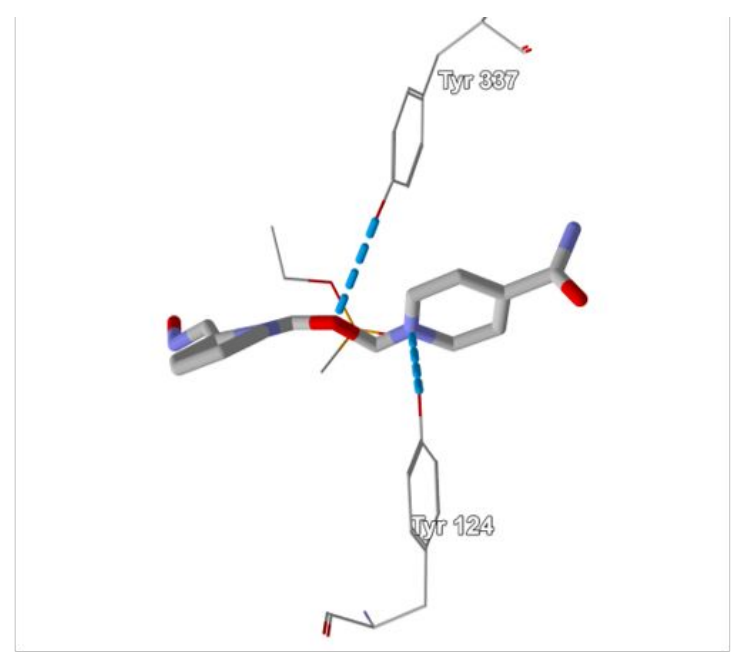

(a)

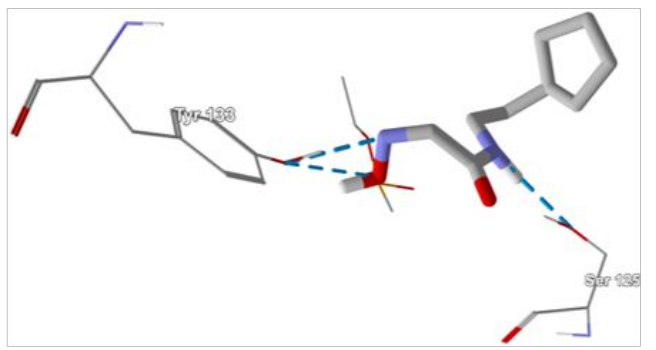

(c)

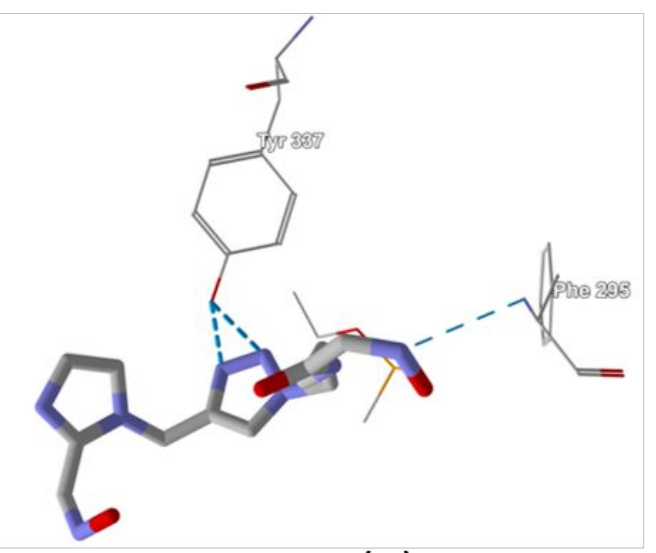

(e)

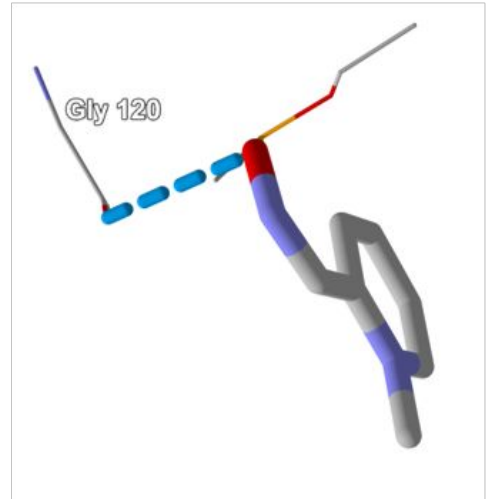

(b)

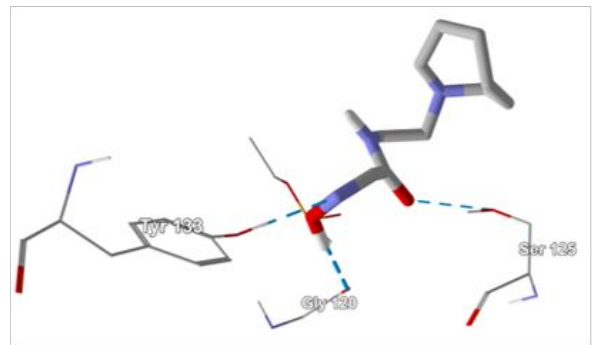

(d)

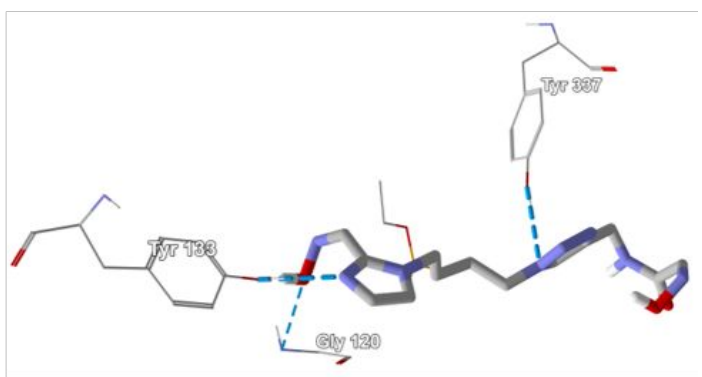

(f)

Figure S2) Oxime interaction modes obtained by docking at the VX inhibited AChE interaction site, highlighting the yellow phosphorus atom in the background. a) $\mathrm{HI}-6$; b) 2-PAM; c) RS41A; d) RS194B; e) RS48B; and f) RS182A. 


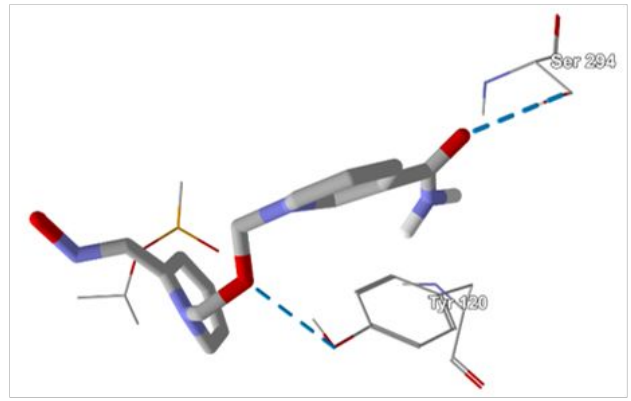

(a)

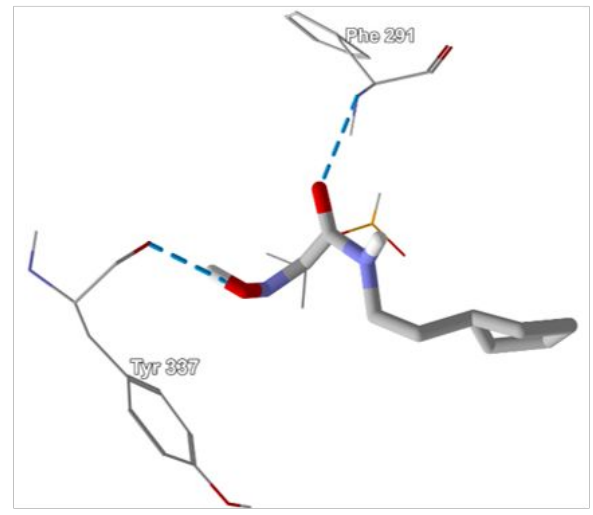

(c)

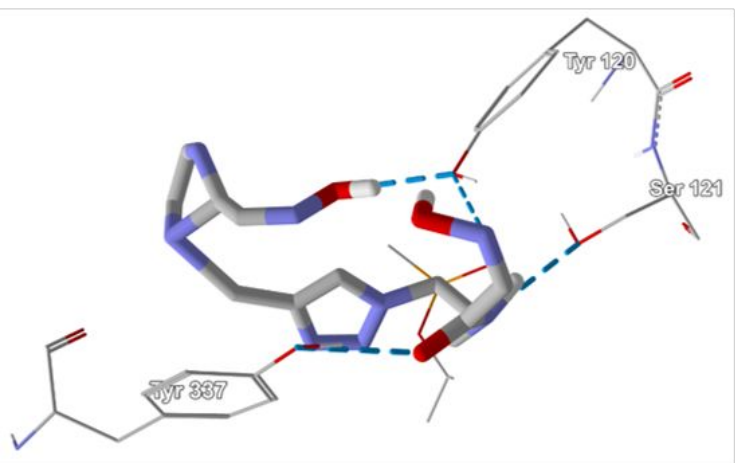

(e)

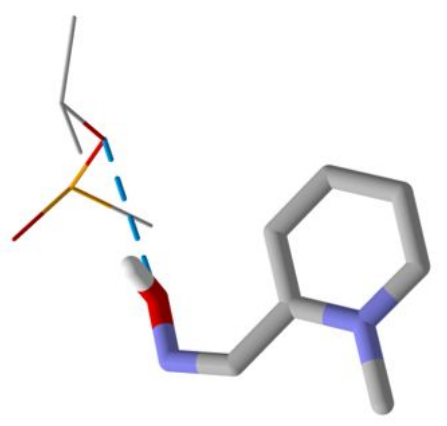

(b)

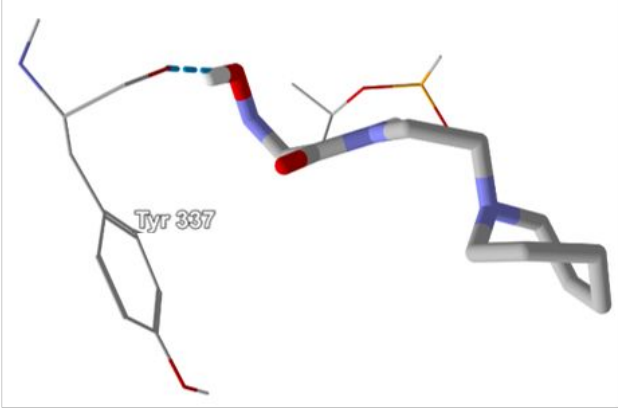

(d)

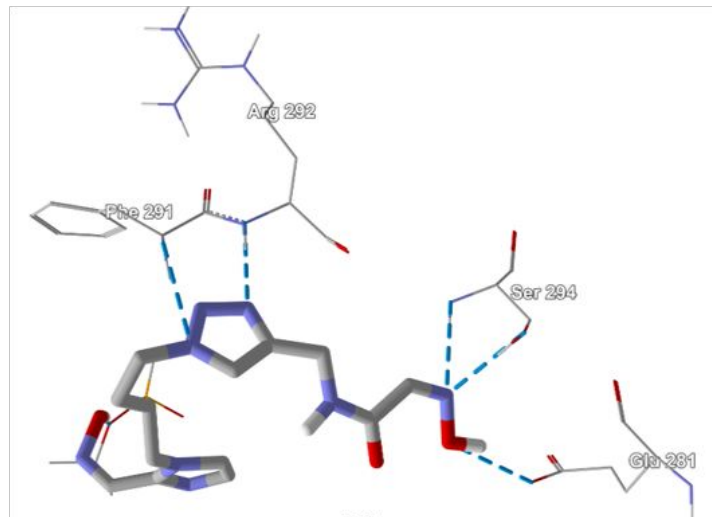

(f)

Figure S3) Oxime interaction modes obtained by docking at the GB inhibited AChE interaction site, highlighting the yellow phosphorus atom in the background. a) $\mathrm{HI}-6$; b) 2-PAM; c) RS41A; d) RS194B; e) RS48B; and f) RS182A. 


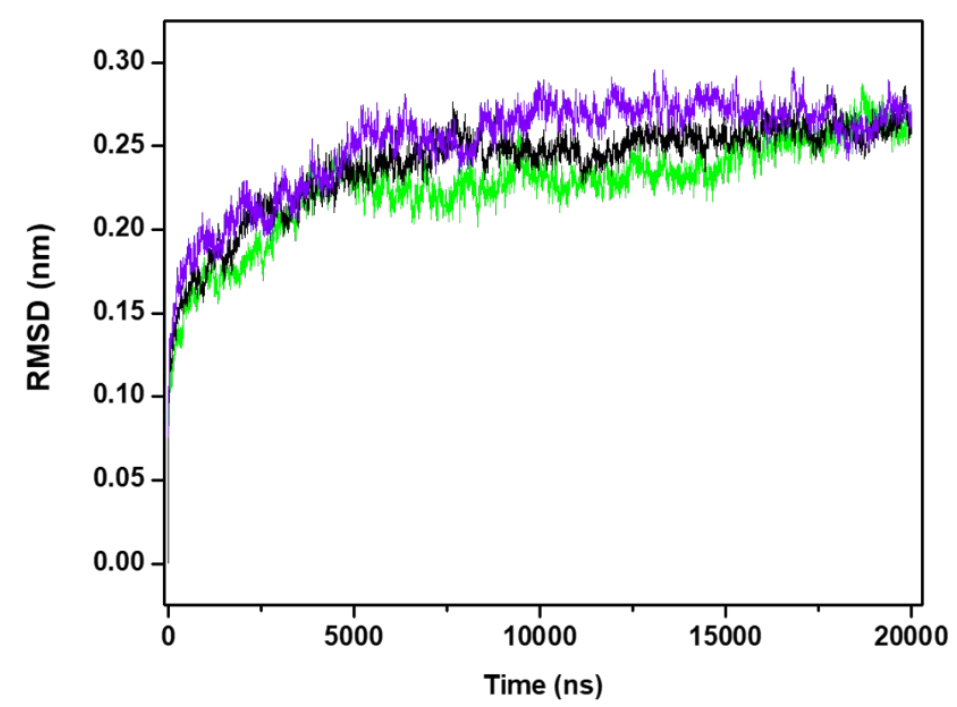

Figure S4) RMSD versus time for apo systems. AChE-GB (green), AChE-VX (black), and AChE (purple).

\section{Distance of COM}

From the histograms of distance between the COM of oxime and OP (Figure S4) it is possible to see the influence of the volume of the interaction site on the stability. As AChE-GB has a larger interaction site, it allows for a displacement of the oximes, causing the distance of the COM to focus on values greater than $1 \mathrm{~nm}$. 


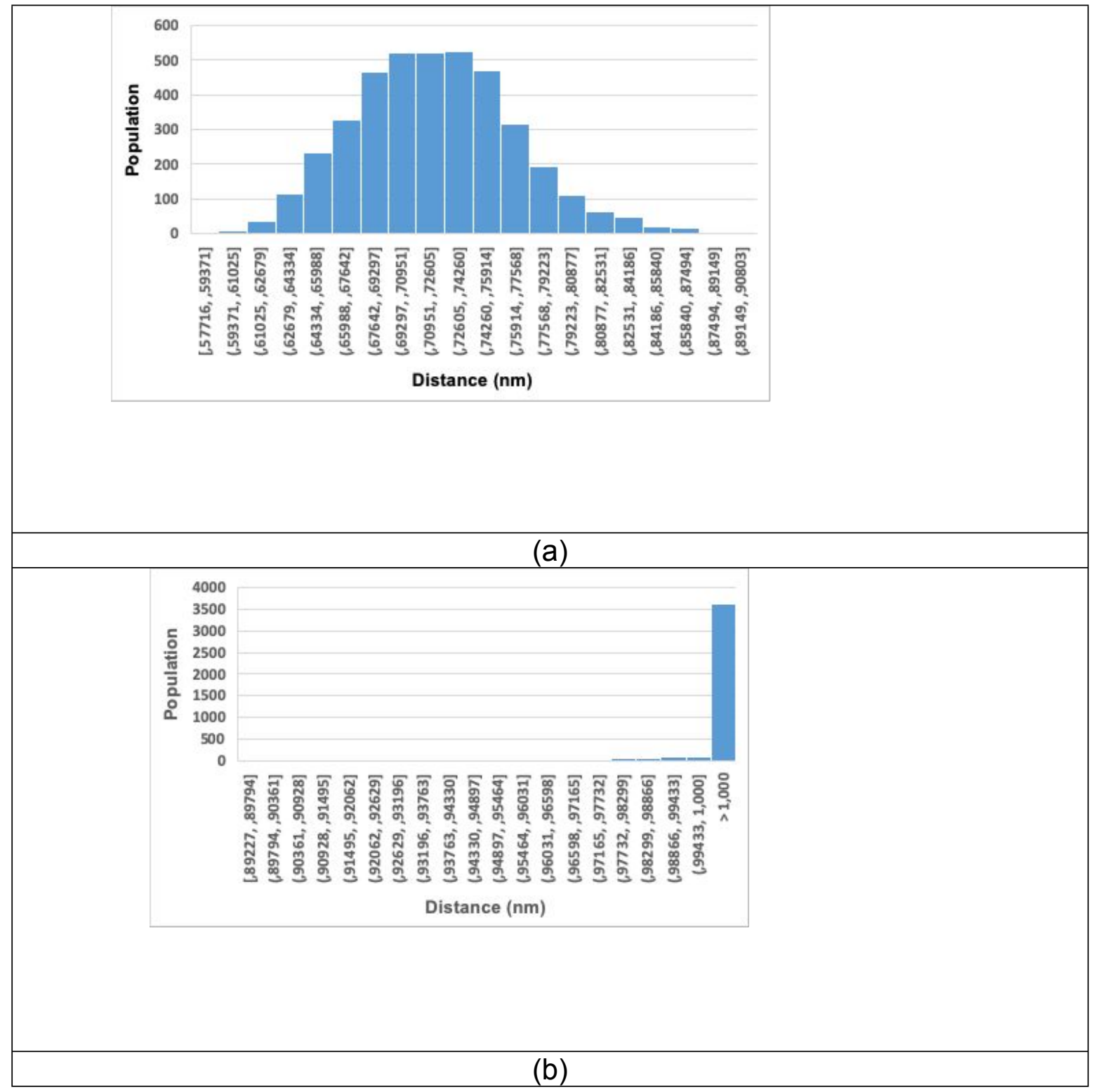




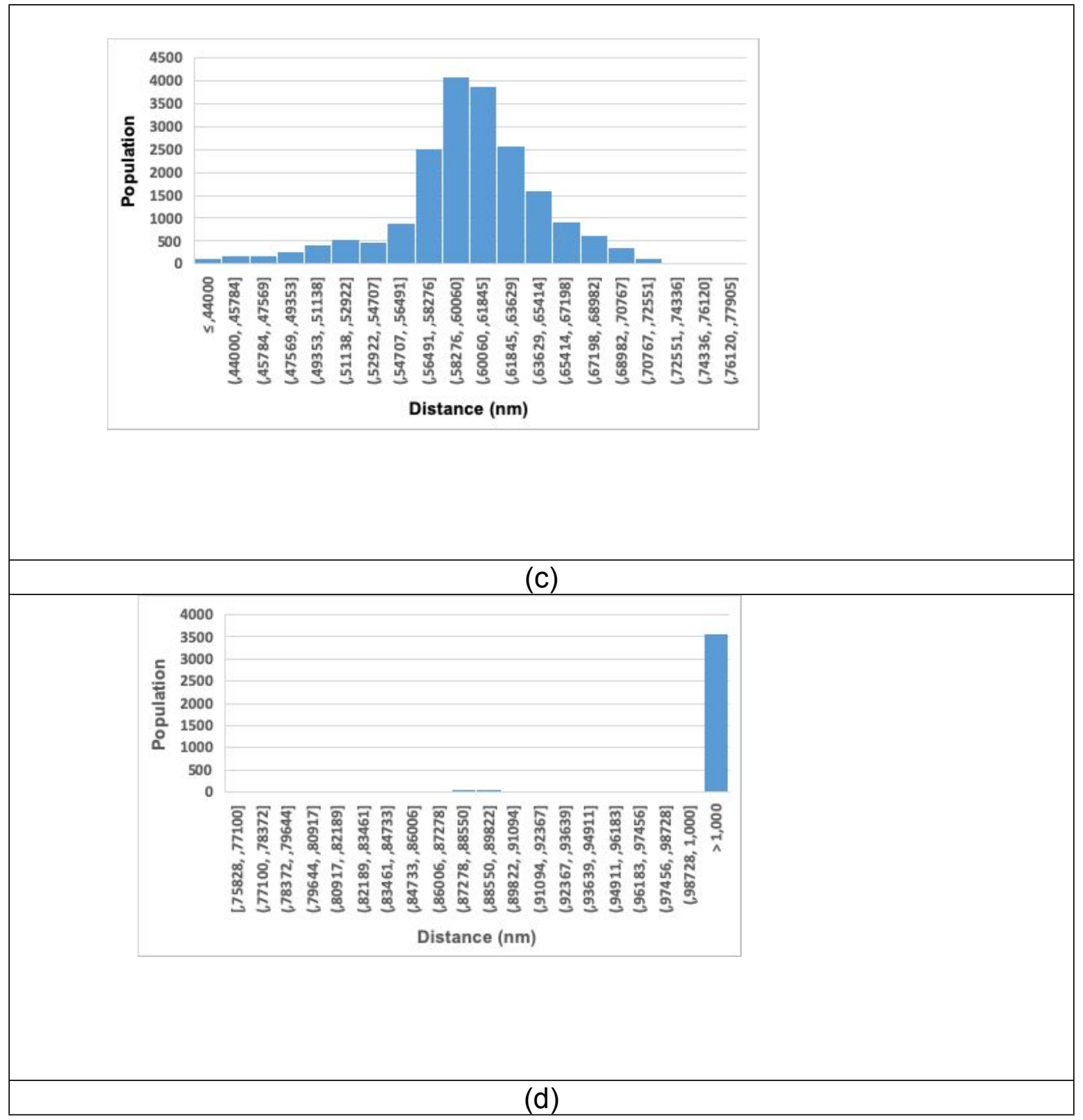




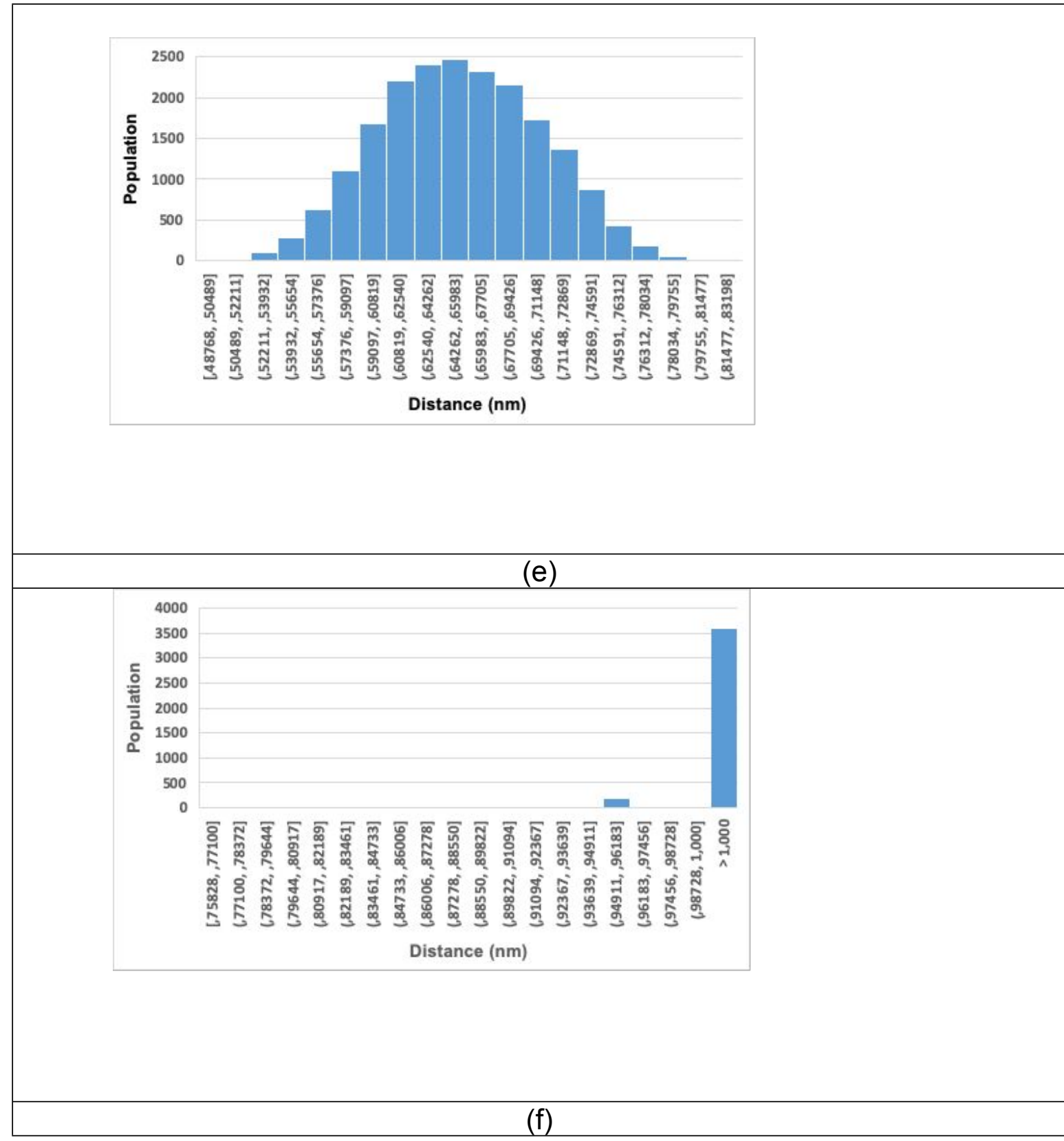

Figure S5) Population histogram of distances between the COM of oxime and OP OP presented during MD simulaton and determined by interactions between oximes and AChE-OP. (a) VX/RS194B, (b) GB/RS194B, (c) VX/48B, (d) GB/RS48B, (e) VX/RS182A, and (f) GB/RS182A. 